\title{
25 Secondary plant compounds
}

Besides the commonly known main ingredients, our nutrients and luxury foods often contain many other bioactive compounds (-Tab. 25.1). The number of individual compounds is estimated in the range of 60,000-100,000. The phytochemicals present in the various types of fruits and vegetables have formed over the millennia of evolution to protect plants against UV radiation, pests and dysregulation during their growth.

Secondary plant compounds only occur in trace amounts and are mostly localized in peels and seeds. They are not temperature-sensitive and therefore made more digestible by boiling or cooking. Since ancient times, people have lived on a regular diet consisting of a broad spectrum of such bioactive plant compounds and thereby optimized their nutritional intake. In a mixed diet, secondary plant compounds, around 10,000 of which we consume regularly, make up approximately $1.5 \mathrm{~g}$ of our daily nutrition.

Examples of these important nutrients include the flavonoids in general and the flavanols in particular, which belong to one of 9 flavonoid subgroups. Some flavanols of note include the antioxidants epicatechin and epigallocatechin gallate. Both occur in tea, cocoa and in many types of fruit. Flavanols are inversely associated with incident type 2 diabetes (Zamora-Ros et al. 2014), they slow down arteriosclerotic processes by limiting the range of motion of smooth muscle cells within the vessel wall, inhibit platelet function and lower blood pressure by blocking the formation of endothelin, a potent vasoconstrictor. The anthocyanidins, another subgroup of the flavonoids, also have an antihypertensive action. They give pigment to the blue, purple and red fruits. Their positive effect on blood pressure was shown in a 14-year prospective
- Tab. 25.1 Important secondary plant substances

\begin{tabular}{|l|l|}
\hline Substance group & Main effects \\
\hline Carotinoids & $1,3,6,8$ \\
\hline Glucosinolates & $1,2,6$ \\
\hline Monoterpenes & 1,2 \\
\hline Phytosterols & 1,6 \\
\hline Protease inhibitors & 1,3 \\
\hline Saponins & $1,2,6,7,8$ \\
\hline Sulfides & $1,2,3,4,5,6,7,8$ \\
\hline Flavonoids & $1,2,3,4,5,6,7,8$ \\
\hline \begin{tabular}{l} 
Phenolic acids \\
\hline Phytoestrogens
\end{tabular} & $1,2,3$ \\
\hline \begin{tabular}{l} 
1 antitumor, 2 antibiotic, 3 antioxidant, \\
4 anticoagulant, 5 blood pressure regulating, \\
6 cholesterol-lowering, 7 anti-inflammatory, \\
\hline immuno-stimulating
\end{tabular} & \\
\hline
\end{tabular}

study on 156,962 participating women and men (Cassidy et al. 2011).

Despite the rapidly growing body of knowledge on a diverse range of plant compounds, which usually only release their health-promoting properties in complex mixtures, they have not been administered for the targeted treatment of specific diseases thus far. During certain drug-based interventions, it is important for patients to avoid furanocoumarin derivatives present in grapefruit because interactions with their breakdown products can change the bioavailability of over 80 drugs in the body (Bailey et al. 2013, Pirmohamed 2013). 